\title{
Digital image processing algorithms for automated inspection of dynamic effects in roller bearings
}

Bettina Altmann, Christian Pape, Eduard Reithmeier

Bettina Altmann, Christian Pape, Eduard Reithmeier, "Digital image processing algorithms for automated inspection of dynamic effects in roller bearings," Proc. SPIE 10334, Automated Visual Inspection and Machine Vision II, 103340A (26 June 2017); doi: 10.1117/12.2269065

SPIE. Event: SPIE Optical Metrology, 2017, Munich, Germany 


\title{
Digital image processing algorithms for automated inspection of dynamic effects in roller bearings
}

\author{
Bettina Altmann ${ }^{\mathrm{a}}$, Christian Pape ${ }^{\mathrm{a}}$, and Eduard Reithmeier ${ }^{\mathrm{a}}$ \\ ${ }^{a}$ Institute of Measurement and Automatic Control, Leibniz Universität Hannover, Nienburger \\ Str. 17, 30167 Hannover, Germany
}

\begin{abstract}
Unstable movement in roller bearings like cage or roller slip can lead to damages or eventually even to an early break of the bearing. To prevent slip, inadequate operating states should be avoided. Therefore, it is necessary to study the dynamic behavior of the bearing. Unfortunately, there is only a limited range of measurement methods for the dynamic of bearing components. Two possible approaches are using solely a high-speed camera or the combination of an optomechanical image derotator and a high-speed camera. This work focuses on a proposal which is suitable for both. Initially, the influence of the rotational velocity in the images is eliminated. In the next step the measurement data is reduced to a region of interest which displays a particular rolling-element. A rolling element is equipped with a linear marker which, in the next stage, is segmented by a thresholding method to multiple regions. The region representing the marker is extracted from the background and the position is calculated by a Principle Component Analysis. Depending on the shift of the angular position and the lag time between two images, the rotational velocity of the rolling element is calculated. Thus, it is possible to determine whether the rolling element is operating under ideal conditions. In conclusion, it can be said that this approach enables a simple and flexible non-invasive method to depict the occurrence of roller slip in roller bearings.
\end{abstract}

Keywords: roller bearings, dynamic behavior analysis, roller slip, image derotator, image processing

\section{INTRODUCTION}

Roller bearings can be found in a wide range of mechanical engineering applications. The performance of the bearing is directly linked to the capability of the entire machine. Unstable movement in the bearing elements (rolling elements, cage, inner/outer race) leads to fatigue and damages or even a reduced lifetime and an early break of the bearing. Talking about roller bearing damages, there are a lot of symptoms like skidding, wear marks, discoloration or cracks with a lot of causes like slip, excessive loading, insufficient lubrication or tight fits. ${ }^{1-3}$ Skidding manifests in spotted smear marks or roughness on the surface of rolling elements or the raceways. ${ }^{4,5}$ Eventually skid damage could lead to cracks and the total destruction of the bearing. Skid damage is caused by poor lubrication which can be induced by slip or other factors such as vibrations or alternating load of the bearing. ${ }^{6-9}$ This work concentrates on slip, which Boness ${ }^{10}$ and Tassone ${ }^{5}$ described as a relative difference in velocity between the rolling-elements of the bearing. Under ideal conditions, all rolling-elements should be operating under pure rolling behavior. In case the radial load on the roller bearing is insufficient to develop an acceptable frictional force between the rolling-element and the raceway, roller slip takes place.

To prevent cage or roller slip, inadequate operating states should be avoided. Therefore, it is necessary to study the dynamic effects in the bearing. Up to now, dynamic effects in roller bearings are not completely understood. To do so, several theoretical studies and simulations have been performed. ${ }^{11-18}$ However, there is only a limited number of measurement methods for a movement analysis of bearing components. Kesler ${ }^{19}$ measured rotational slip of the inner race of a railroad bearing by detecting installed magnets on the axle and inner race using hall sensors. Royzman ${ }^{20}$ investigated the cage slip in testing contact and contactless (inductive/capacitive sensing) methods. Sakaguchi, ${ }^{17,18}$ Evans $^{9}$ and $W^{21}{ }^{21}$ used eddy current sensors to measure displacements of the cage. Though, these approaches are only suitable for metal cages or cages with adhesive

Further author information:

E-mail: bettina.altmann@imr.uni-hannover.de, Telephone: +49 5117624280 
metal tape. All those methods do not provide information about characteristics of the rolling elements and influence the properties of the bearing due to the changes of the components mentioned above. Selvaraj ${ }^{22}$ analyzed the cage and roller slip of cylindrical roller bearings in a newly designed test rig. He modified the rolling elements so they transmit power to planet gears by a square rod. In measuring the velocity of the slip preventing gear he determines the speed of the cage and the rolling elements indirectly. Abele ${ }^{23}$ and Yang $^{24}$ abstain from changing the components of the bearing and use a high-speed camera and image processing algorithms to investigate the movement of the bearing cage but still provide no information about the rolling elements. Reithmeier et al. ${ }^{25}$ developed an optomechanical image derotator, which Mirzaei ${ }^{26}$ applied in combination with a high-speed camera to measure slip of the cage and rolling elements. The derotator operates in tracking the rotation of the measurement object so that quasi-stationary images are acquired through multiple reflections of the bearing by a mirror assembly placed inside the derotator. In doing this, the rotational velocity of the cage is eliminated in the acquired images so the rotational velocity of the rolling elements can be measured in a convenient way.

This work presents an automated inspection algorithm which is flexibly applicable for using solely a highspeed camera or the combination of an image derotator and a high-speed camera. Initially, images of the roller bearing have to be derotated (either optically by means of the derotator or by use of image processing algorithms) so that the influence of the rotational velocity is eliminated. In case only the camera is used, the image data is rotated via image processing around the center of the bearing. Either way, in the next step the measurement data is reduced to a region of interest which displays a particular rolling-element. This is done by a pre-segmentation of the image to emphasize the rolling-elements and a circular fit to detect them. A rolling-element is equipped with a linear marker which, in the next stage, is segmented and classified by computer vision algorithms by segmenting only the circular region to multiple segments using a thresholding method. The region representing the marker is extracted from the background and the position is calculated by a Principle Component Analysis (PCA). Depending on the shift of the angular position and the lag time between two images, the rotational velocity of the rolling element is calculated. Thus, it is possible to determine whether the rolling element is operating under ideal conditions or roller slip is present.

\section{OPTOMECHANICAL IMAGE DEROTATOR}

The essential part of the image derotator is a reflector assembly placed inside a hollow-shaft synchronous motor. Fig. 1 depicts the reflector, which consist of a reflector prism and an eccentrically positioned reflector both made of aluminum. Its optical path and working mechanism is based on an Abbe-Koenig prism. In aligning the measurement object (the test bearing), the reflector assembly and the image plane of a camera, a standing image can be acquired. Furthermore, the rotational velocity of the test bearing and the derotator have to be synchronized. In case the rotating bearing is observed through the reflector assembly which is rotating with half of the velocity, a rotating point can be viewed stationary in the image plane.

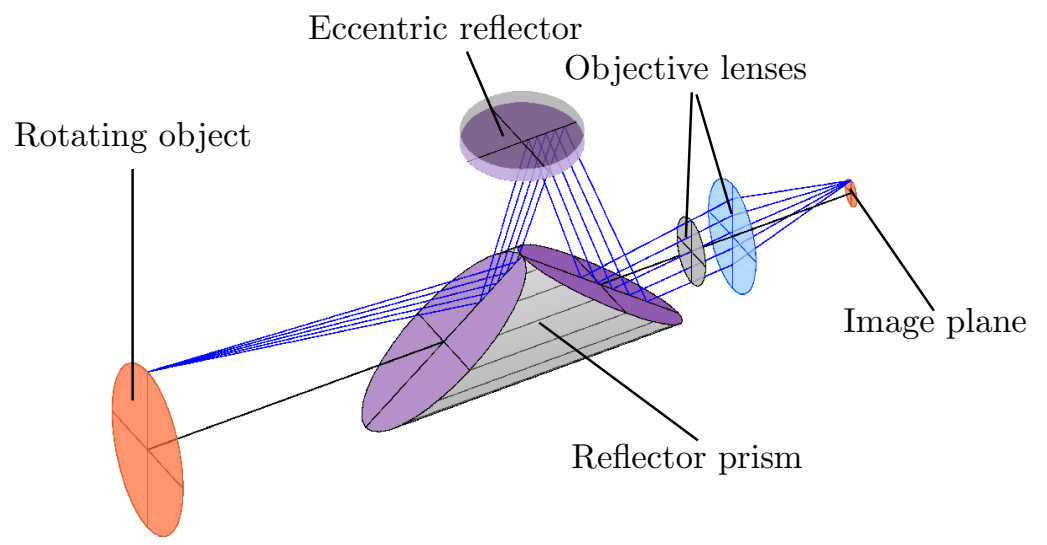

Figure 1: Optical path through the reflector assembly inside the derotator. 
To bring the optical axis of the reflector assembly into line with the rotational axis of the bearing, the derotator is positioned with a parallel kinematic. The adherence of the rotational velocity at a ratio of $1: 2$ is implemented using a controller. Both is not part of the research in this paper.

\section{METHODOLOGY}

To analyze the bearing concerning slip of the rolling elements, the movement of the bearing is acquired by a high-speed camera. A linear marker is applied on the rolling elements (see Fig. 2). The objective is to measure the actual rotational velocity of the rolling elements by detecting the markers on the rolling elements in the captured images by image processing. For this, typical machine vision algorithms are utilized, which are detailed in the following. In comparing the actual velocity of the elements with the predicted velocity from the epicyclical considerations, roller slip is identified.

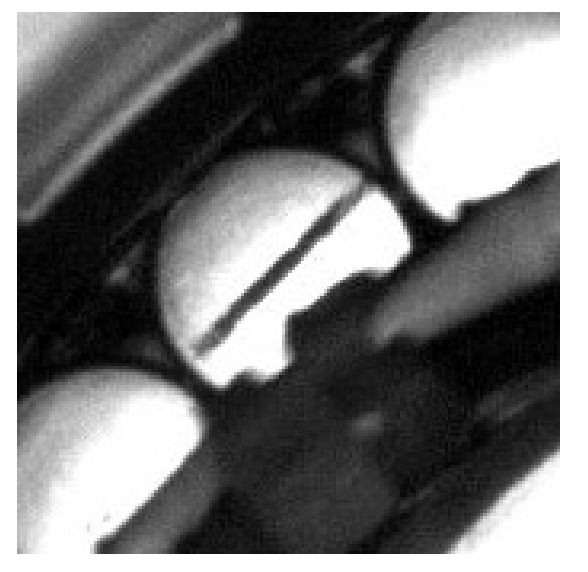

Figure 2: Rolling element with linear marker.

\subsection{Image derotation}

The first step is to eliminate the rotational velocity of the cage $\omega_{c}$. This results in images where the rolling elements are rotating around the same center in the image plane. In doing this the analysis of the rolling elements is made more convenient. In case the measurement data is acquired with the help of the derotator rotating with half of the speed of the cage, this is achieved automatically arising from the operating principle of the derotator. ${ }^{25}$

If the high-speed camera is used exclusively, the rational velocity of the cage $\omega_{c}$ has to be eliminated by image processing. First, the images are displaced, so that the center of the bearing is in the center of the image. In the next step the images are rotated around the image center by

$$
\varphi=\frac{\omega_{c}}{f_{i}}
$$

where $\omega_{c}$ is converted to $\mathrm{deg} \cdot \mathrm{s}^{-1}$ and $f_{i}$ is the frame rate of the high-speed camera in fps.

\subsection{Marker detection}

Afterwards, the images are restricted to a region of interest (ROI) which contains the rolling element to be analyzed. The ROI can be chosen by the user. In the next step the ROI should be further restricted to a circular region containing the rolling element. This is done by a circular Hough transformation. ${ }^{27}$ First, binary images of the ROI have to be achieved by a thresholding method. To apply the Hough transformation the significant edges have to be found using an edge detection algorithm. In doing this an underlying circle structure can be identified in all edge pixels: For each point $(x, y)$ the position of all possible circle centers $\left(x_{c}, y_{c}\right)$ with radius $r$ is calculated by

$$
\left(x-x_{c}\right)^{2}+\left(y-y_{c}\right)^{2}=r^{2}
$$



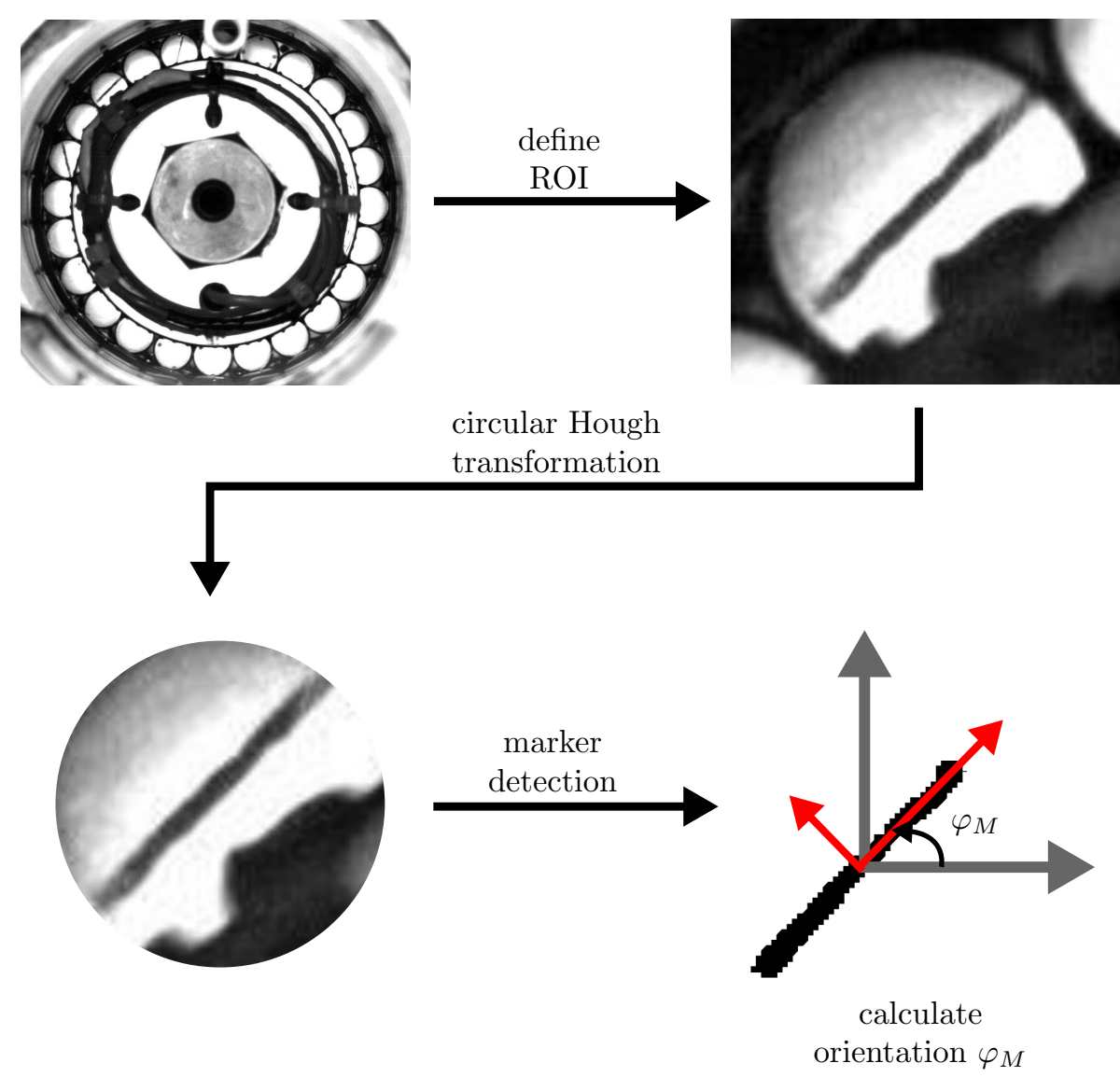

Figure 3: Algorithm for detecting the marker on the rolling elements.

and accumulated in the 3D parameter space $\left(x_{c}, y_{c}, r\right)$. In case the edge points represent a circle structure, this leads to a local maxima in the Hough parameter space. This method enables finding the edge of the circular rolling elements so the ROI can be reduced. In bounding the radii $r$ the results can be further improved. The circular Hough transformation is robust in the face of disturbances and masking of the rolling element.

Subsequently the pixels representing the marker have to be extracted from the remaining ROI. Since the marker is in contrast to the rolling element the first step is to segment the image by a Histogram shape-based thresholding method to create a binary image. In using basic binary morphology operators like opening and closing the quantity of the segments is further reduced so ideally just the background, the rolling element surface and the marker remain. The marker is extracted from the rest by different not specified criteria so the result is a binary image with only the pixels representing the marker.

To determine roller slip, the orientation respectively the angular position of the marker on the rolling element has to be calculated apriori. This is performed by means of a PCA of the remaining pixels. In computing the covariance matrix $\operatorname{Cov}(\mathbf{X})$ of the normalized data points $X$ of the marker and performing a singular value decomposition of $\operatorname{Cov}(\mathbf{X})=U \Sigma V$, the eigenvectors and corresponding eigenvalues can be determined. The eigenvector with the higher eigenvalue is defined as the orientation of the marker. Referred to the $\mathrm{x}$-axis of th Cartesian coordinate system, the orientation of the marker is stated as $\varphi_{M}$. The whole procedure of the algorithm is outlined in Fig. 3. 


\subsection{Slip calculation}

Based on the measurement data acquired with the high-speed camera, the roller slip can be calculated from the data of two consecutive steps $n$ and $n+1$. The roller slip $S_{n}$ in step $n$ is defined as

$$
S_{n}=\frac{\omega_{R, n}-\omega_{R, n}^{\prime}}{\omega_{R, n}} \cdot 100
$$

where $\omega_{R}$ is the rolling element speed in pure rolling condition predicted from the epicyclical considerations and $\omega_{R}^{\prime}$ is the actual velocity measured by means of the marker. The former is calculated by

$$
\omega_{R}=\frac{\omega_{B}}{2} \cdot\left(\frac{d_{p}}{d_{R}}-\frac{d_{R} \cdot \cos ^{2} \alpha}{d_{p}}\right)
$$

where $\omega_{B}$ equals the rotational velocity of the shaft, $\alpha$ equals the contact angle of the bearing, $d_{p}$ equals the pitch diameter and $d_{R}$ equals the rolling element diameter. The latter is determined as

$$
\omega_{R, n}^{\prime}=\delta_{n} \cdot f_{i}
$$

from the change in angle $\delta_{n}=\varphi_{M, n+1}-\varphi_{M, n}$ of the marker's orientation $\varphi_{M}$ in two consecutive steps $n$ and $n+1$. The roller slip $S$ can be plotted over the rotation angle of the bearing afterwards to visualize the location where slip occurs in the bearing.

\section{APPLICATION AND RESULTS}

In the following, the algorithm specified in Sec. 3 is applied to measurement data of the cylindrical roller bearing illustrated in Fig. 3 to proof its functionality. The measurement data is acquired with a CMOS-Camera with a frame rate of $f_{i}=2000 \mathrm{fps}$ and a resolution of $860 \mathrm{px} \times 800 \mathrm{px}$. For the results in Fig. 4a the derotation of the images was performed solely by image processing. Furthermore, the rotational velocity of the shaft is $\omega_{B} \approx 1000 \mathrm{rpm}$ which corresponds to a predicted cage speed of $\omega_{c} \approx 434 \mathrm{rpm}$ and roller speed of $\omega_{R} \approx 2990 \mathrm{rpm}$.

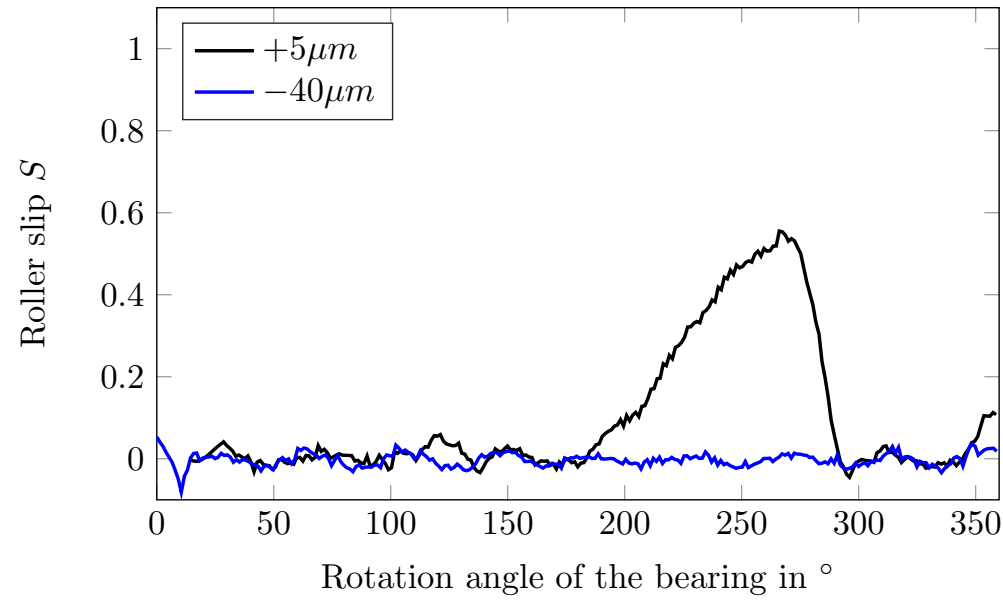

(a) Influence of the radial internal clearance of the bearing on roller slip.

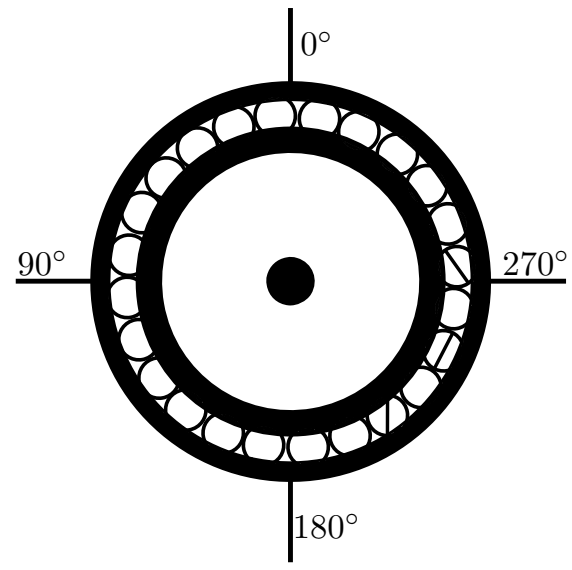

(b) Rotation angle of the bearing.

Figure 4: Results of measurements on roller slip.

Fig. 4a shows the results of two different series of measurements. They vary in the radial internal clearance, so one has a positive $(+5 \mu \mathrm{m})$ and the other a negative clearance $(-40 \mu \mathrm{m}) . \quad S=0$ stands for no occurrence of roller slip, whereas $S=1$ means a pure sliding movement of the rolling element. The rotation angle of the bearing is declared in Fig. 4b.

According to the data in Fig. 4a, a negative clearance has a positive impact on the operating characteristics of the bearing since no roller slip occurs. A positive clearance leads to roller slip at rotation angles between 
$180^{\circ}$ to $300^{\circ}$. As Mirzaei ${ }^{26}$ states, the load zone of the bearing is located at around $100^{\circ}$ to $260^{\circ}$ of the bearing. There the force on the rolling elements is increasing. When the rolling elements leave the load zone they are slowed down because transmission between them and the inner ring is decreased. This leads to slip of the rolling elements.

\section{CONCLUSION}

The analysis of dynamic effects in roller bearings is essential to understand their operating principle and guarantee proper functioning. This paper introduced an algorithm to examine roller bearings. In conclusion, it can be said that this approach enables a simple and flexible non-invasive method to quantify the occurrence of roller slip in roller bearings. Thus, the influence of various factors on slip like internal clearance, rotational velocity, loads or the lubricant can be investigated. Furthermore, the algorithm could be extended to analyze even more dynamic effects which influence the operating behavior of a roller bearing. More experiments on measuring the cage slip will be implemented hereinafter in future work.

\section{REFERENCES}

[1] Gupta, P. K., "Transient Ball Motion and Skid in Ball Bearings," Journal of Lubrication Technology 97(2), 261 (1975).

[2] Gupta, P. K., [Advanced Dynamics of Rolling Elements], Springer, New York (1984).

[3] Jacobs, G. and Plogmann, M., "Rolling Bearing Damages," in [Encyclopedia of Lubricants and Lubrication], Mang, T., ed., 1600-1618, Springer Berlin Heidelberg, Berlin, Heidelberg (2014).

[4] Harris, T. A., "An Analytical Method to Predict Skidding in High Speed Roller Bearings," ASLE Transactions 9(3), 229-241 (1966).

[5] Tassone, B. A., "Roller bearing slip and skidding damage," Journal of Aircraft 12(4), 281-287 (1975).

[6] Kacmarsky, W. M. and Hewko, L. O., "Effect of a High Traction Fluid on Skidding in a High Speed Roller Bearing," Journal of Lubrication Technology 93(1), 11 (1971).

[7] Poplawski, J. V., "Slip and Cage Forces in a High-Speed Roller Bearing," Journal of Lubrication Technology 94(2), 143 (1972).

[8] Hamer, J. C., Sayles, R. S., and Ioannides, E., "An Experimental Investigation Into the Boundaries of Smearing Failure in Roller Bearings," Journal of Tribology 113(1), 102 (1991).

[9] Evans, R. D., Barr, T. A., Houpert., L., and Boyd, S. V., "Prevention of Smearing Damage in Cylindrical Roller Bearings," Tribology Transactions 56(5), 703-716 (2013).

[10] Boness, R. J., "Cage and roller slip in high-speed roller bearings," Journal of Mechanical Engineering Science 11(2), 181-188 (1969).

[11] Gupta, P. K., "Frictional Instabilities in Ball Bearings," Tribology Transactions 31(2), 258-268 (1988).

[12] Gupta, P. K., "Modeling of Instabilities Induced by Cage Clearances in Ball Bearings," Tribology Transactions 34(1), 93-99 (1991).

[13] Ye, Z. H. and Wang, L. Q., "Cage Instabilities in High-Speed Ball Bearings," Applied Mechanics and Materials 278-280, 3-6 (2013).

[14] Stacke, L. E. and Fritzson, D., "Dynamic behaviour of rolling bearings: Simulations and experiments," Proceedings of the Institution of Mechanical Engineers, Part J: Journal of Engineering Tribology 215(6), 499-508 (2001).

[15] Pederson, B. M., Sadeghi, F., and Wassgren, C., "The Effects of Cage Flexibility on Ball-to-Cage Pocket Contact Forces and Cage Instability in Deep Groove Ball Bearings," in [SAE 2006 World Congress 83 Exhibition], SAE Technical Paper Series, SAE International400 Commonwealth Drive, Warrendale, PA, United States (2006).

[16] Ghaisas, N., Wassgren, C. R., and Sadeghi, F., "Cage Instabilities in Cylindrical Roller Bearings," Journal of Tribology 126(4), 681 (2004).

[17] Sakaguchi, T. and Harada, K., "Dynamic Analysis of Cage Behavior in a Tapered Roller Bearing," Journal of Tribology 128(3), 604 (2006). 
[18] Sakaguchi, T. and Harada, K., "Dynamic Analysis of Cage Stress in Tapered Roller Bearings Using Component-Mode-Synthesis Method," Journal of Tribology 131(1), 011102 (2009).

[19] Kesler, J. K., Stuart, C., and Gray, D. E., "Measurement of the rotational slippage of the inner race of railroad bearings," in [Nondestructive Evaluation of Aging Infrastructure], Gray, D. E. and Stone, D. H., eds., SPIE Proceedings, 128-136, SPIE (1995).

[20] Royzman, V. P. and Dobronos, L., "Measuring of Slipping in Rolling Bearings," IMAC-XX: Conference $\mathcal{E}$ Exposition on Structural Dynamics (2002).

[21] Wen, B., Ren, H., Zhang, H., and Han, Q., "Experimental investigation of cage motions in an angular contact ball bearing," Proceedings of the Institution of Mechanical Engineers, Part J: Journal of Engineering Tribology 100, 1-15 (2017).

[22] Selvaraj, A. and Marappan, R., "Experimental analysis of factors influencing the cage slip in cylindrical roller bearing," The International Journal of Advanced Manufacturing Technology 53(5-8), 635-644 (2011).

[23] Abele, E. and Holland, L. Nehrbass, A., "Image Acquisition and Image Processing Algorithms for Movement Analysis of Bearing Cages," Journal of Tribology 138(2), 1-7 (2016).

[24] Yang, Z., Chen, H., Yu, T., and Li, B., "A high-precision instrument for analyzing nonlinear dynamic behavior of bearing cage," The Review of scientific instruments 87(8), 085105 (2016).

[25] Reithmeier, E., Mirzaei, S., and Kasyanenko, N., "Optical vibration and deviation measurement of rotating machine parts," Optoelectronics Letters 4(1), 45-48 (2008).

[26] Mirzaei, S., Fahlbusch, T., Reithmeier, E., and Poll, G., "Experimental slip measurement of roller bearings," 2nd Ph.D. Conference AI4IA, Hannover, Germany (2008).

[27] Atherton, T. J. and Kerbyson, D. J., "Size invariant circle detection," Image and Vision Computing 17(11), 795-803 (1999). 